\title{
Influence of Surface Roughness on Evaluation of Stress Gradients in Coatings
}

\author{
A.Benediktovich ${ }^{1, a}$, H.Guerault ${ }^{2}$, I.Feranchuk ${ }^{1, b}$, \\ V.Uglov ${ }^{1}$ and A.Ulyanenkov ${ }^{2, c}$ \\ ${ }^{1}$ Belarusian State University, 4 Nezavisimosti Av., 220030, Minsk, Belarus \\ ${ }^{2}$ Bruker AXS GmbH, Östliche Rheinbrückenstr. 49, 76187, Karlsruhe, Germany \\ aandrey.benediktovich@bruker-axs.by, bilya.feranchuk@bruker-axs.by, \\ calex.ulyanenkov@bruker-axs.de
}

Keywords: residual stress gradient, roughness, GIXRD

\begin{abstract}
Roughness influence on the residual stress gradient evaluation in the case of a grazing incidence X-ray diffraction setup is considered. In this geometry the surface roughness changes essentially the X-ray wave fields of the transmitted and diffracted beams inside the coatings and subsurface regions of bulk samples, and thus influences the refractive properties of the investigated sample area. In turn, the change in the refraction index enforces the re-scale of the informational depth and, consequently, the evaluated stress depth profile. The diffracted amplitude from the crystalline grain located beneath the surface is calculated. The surface roughness is shown to contribute into reconstruction of the real stress gradient profile of the coating.
\end{abstract}

\section{Introduction}

One of the measurement strategies for non-destructive analysis of the residual stress gradient is based on the experimental setup corresponding to the grazing incidence X-ray diffraction (GIXRD) [1]. In this case the penetration depth $\tau$ and refraction index $n$ strongly depend on the angles both for the incident and diffraction beams and it leads to sensitivity of the Bragg peak position to the normal coordinate of the scattering grain. Commonly used theoretical treatments $\tau$ and $n$ assume an ideal flat surface [1], whereas experimental investigations indicate the influence of roughness on the stress evaluation results [2]. Therefore, the theoretical analysis of this effect is of great interest for applications, and especially for coatings.

Let us consider the qualitative estimate of the characteristic parameters of the problem. Fig.1 shows sketch of the beam traces when $X$-rays are scattered by the grain with the center located on the depth $Z_{\mathrm{i}}$ under sample surface. Roughness with the averaged amplitude $u$ changes the electron density in the transition layer with the characteristic thickness $\sim u$. Thus, the phases of the waves scattered by the grain and defining the Bragg peak position could be sensitive to the roughness when $\mathrm{Z}_{\mathrm{i}} \sim u$. From the other side, diffraction from the grain could be observable if $\mathrm{Z}_{\mathrm{i}}$ is less than the effective penetration depth which is defined by the formula [3]:

$$
\tau=\frac{1}{\mu} \frac{\sin \alpha \sin \beta}{\sin \alpha+\sin \beta},
$$

where $\mu$ is the linear absorption coefficient. In the GIXRD geometry $\alpha<<1$, and the roughness could be important for the stress evaluation if ( $\alpha$ is in radian units)

$$
u \mu \geq \alpha
$$




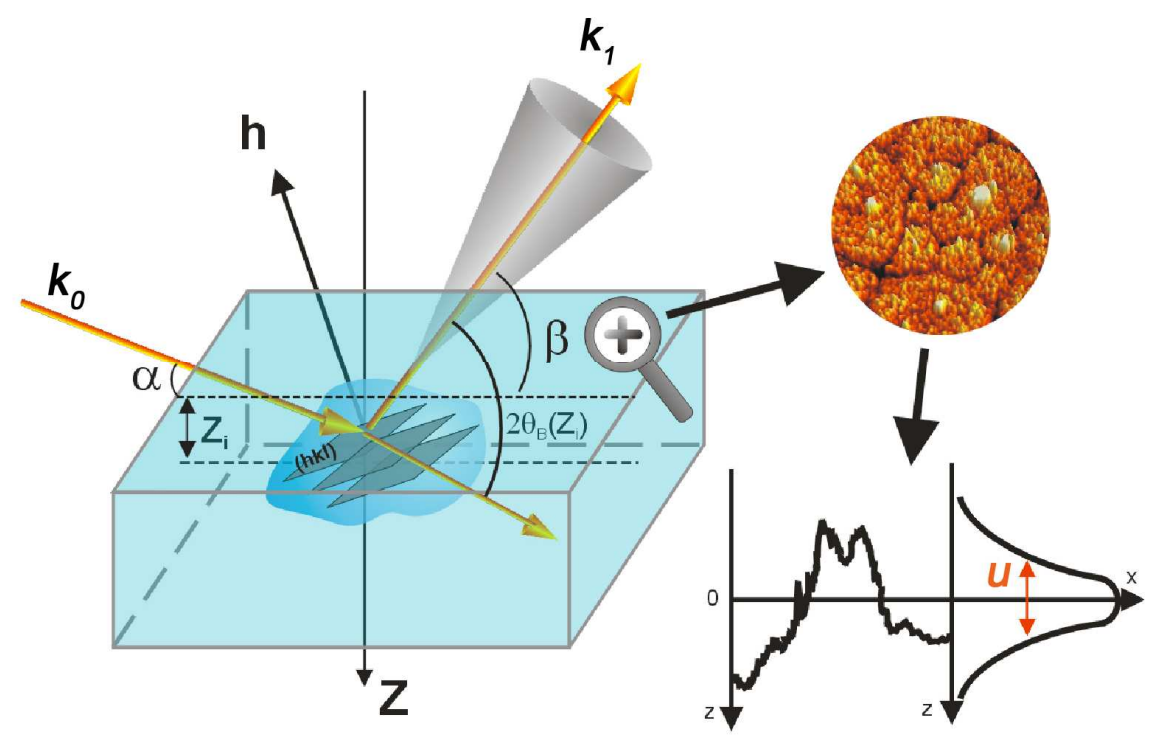

Figure 1

Sketch of the X-ray diffraction from the grain on the depth $\mathrm{Z}_{\mathrm{i}}$ in a sample: $\mathbf{k}_{0}$ and $\mathbf{k}_{1}$ are the incident and scattered wave vectors; $\mathbf{h}$ is the reciprocal lattice vector corresponding to Bragg angle $\theta\left(Z_{\mathrm{i}}\right) ; \alpha$ and $\beta$ are the incident and exit angles; $u$ is the root mean square of the Gauss roughness distribution.

\section{Calculation of the diffraction intensity}

Distorted wave Born approximation (DWBA) is further used for the rigorous analysis, which permits to take into account the deviations of the incident and diffracted beams from the plane waves due to scattering in the rough transition layer. In this case, the differential scattering cross section for the grain with volume $\mathrm{V}_{\mathrm{i}}$ centered in the point $\mathrm{Z}_{\mathrm{i}}$ is defined by the following expression [4]:

$$
\frac{d I\left(Z_{i}\right)}{d \Omega}=\frac{k_{0}^{4}\left(1+\cos ^{2} \theta_{B}\right)}{32 \pi^{2}}\left|\chi_{h}\right|^{2}\left|\int_{V i} E_{f}^{*}\left(\mathbf{r}+\mathbf{N} Z_{i}\right) \exp \left[i \mathbf{h}\left(Z_{i}\right) \mathbf{r}\right] E_{i}\left(\mathbf{r}+\mathbf{N} Z_{i}\right) d \mathbf{r}\right|^{2}
$$

Here $\mathbf{N}$ is the internal normal to the surface; $\mathrm{k}_{0}$ is the absolute value of $\mathrm{x}$-ray radiation wavevector in vacuum, $\theta_{B}$ is a Bragg angle, $\chi_{h}$ is the Fourier component of the grain X-ray polarizability and it is supposed that the grains are distributed homogeneously in the lateral plane; $E_{i}$ and $E_{f}$ are the incident and diffracted wave fields with arbitrary polarization. These fields should be found as the solutions of the Maxwell equations

$\left[\Delta+\mathrm{U}(\mathbf{r})+\mathrm{k}_{0}^{2}\right] \mathrm{E}(\mathbf{r})=0$

where the scattering potential $\mathrm{U}(\mathbf{r})=\mathrm{k}_{0}^{2} \chi_{0} \mathrm{H}\left[\mathrm{z}-\mathrm{z}_{0}(\mathbf{r})\right]$ depending on the rough surface form $\mathrm{z}_{0}(\mathbf{r})$; $\mathrm{H}(\mathrm{z})$ is the Heaviside step function. This form of scattering potential enables to take into account effects of roughness and refraction simultaneously which are significant at small angles close to the angle of total reflection. When the scattering potential is averaged over the roughness, it is split into the coherent $\left(\mathrm{U}_{\mathrm{c}}\right)$ and diffuse $\left(\mathrm{U}_{\mathrm{d}}\right)$ parts. Diffuse scattering does not affect the Bragg peak shift and $\mathrm{U}_{\mathrm{c}}(\mathrm{z})$ defines the form of the interface transition layer. If the Gaussian distribution function is used for roughness, we obtain [4]: 


$$
\mathrm{U}_{\mathrm{c}}(\mathrm{z})=\mathrm{k}_{0}^{2} \chi_{0} \lambda(\mathrm{z}) ; \quad \lambda(\mathrm{z})=\frac{1}{\sigma \sqrt{2 \pi}} \int_{-\infty}^{\mathrm{z}} \exp \left[-\frac{\mathrm{t}^{2}}{2 \mathrm{u}^{2}}\right] \mathrm{dt}
$$

Approximate analytical solution of Eq. 4 with the potential from Eq. 5 was found in [5]. For example, the incident wave field has the following form:

$$
\begin{aligned}
& \mathrm{E}_{\mathrm{i}}(\mathbf{r})=\mathrm{A}_{\mathrm{i}}(\mathrm{z}) \exp \left[\mathrm{ik}_{\perp} \mathbf{r}_{\perp}\right] ; \quad \mathrm{A}_{\mathrm{i}}(\mathrm{z})=\left[\mathrm{e}^{\mathrm{iqz}}+\mathrm{R}(\mathrm{q}) \mathrm{e}^{-\mathrm{iqz}}\right][1-\lambda(\mathrm{z})]+\mathrm{T}(\mathrm{q}) \mathrm{e}^{\mathrm{ipz}} \lambda(\mathrm{z}) \\
& \mathrm{q}=\mathrm{k}_{0} \sin \alpha ; \mathrm{p}=\mathrm{k}_{0} \sqrt{\sin ^{2} \alpha+\chi_{0}} ; \mathrm{R}=\frac{\mathrm{q}-\mathrm{p}}{\mathrm{q}+\mathrm{p}} \exp \left(-2 \mathrm{qpu}^{2}\right) ; \mathrm{T}=\frac{2 \mathrm{q}}{\mathrm{q}+\mathrm{p}}
\end{aligned}
$$

Diffracted wave field is defined by the analogous expression with the corresponding indices.

Total diffraction intensity from the whole sample is calculated by the summation on $Z_{\mathrm{i}}$ that transforms to the integral if the grains are distributed homogeneously with the linear density $\zeta$ :

$$
<\frac{\mathrm{dI}}{\mathrm{d} \Omega} .>=\mathrm{Q} \int_{0}^{\mathrm{t}}\left|\mathrm{A}_{\mathrm{i}}(\mathrm{z})\right|^{2}\left|\mathrm{~A}_{\mathrm{f}}(\mathrm{z})\right|^{2} \Phi\left[\theta-\theta_{\mathrm{B}}(\mathrm{z})\right] \mathrm{dz} ; \quad \mathrm{Q}=\zeta\left|\chi_{\mathrm{h}}\right|^{2} \frac{\mathrm{k}_{0}^{4}\left(1+\cos ^{2} \theta_{\mathrm{B}}\right)}{32 \pi^{2}}
$$

Here $t$ is thickness of the sample and function $\Phi\left[\theta-\theta_{\mathrm{B}}(\mathrm{z})\right]$ describes the peak shape centered at the angle $\theta_{\mathrm{B}}(\mathrm{z})$. As usual, the peak shift $\Delta \theta=\left[\theta_{\mathrm{B}}(\mathrm{z})-\theta_{\mathrm{B} 0}\right]$ relative to the position $\theta_{\mathrm{B} 0}$ for free crystal defines the strain profile $\sigma_{\mathrm{ij}}(\mathrm{z})[6]$. The expression for the measured profile $\sigma_{\mathrm{ij}}(\tau, \mathrm{u})$ can be found using the same arguments as in the paper [7]:

$\sigma_{\mathrm{ij}}(\tau, \mathrm{u})=\frac{\int_{0}^{\mathrm{t}}\left|\mathrm{A}_{\mathrm{i}}(\mathrm{z})\right|^{2}\left|\mathrm{~A}_{\mathrm{f}}(\mathrm{z})\right|^{2} \sigma_{\mathrm{ij}}(\mathrm{z}) \mathrm{dz}}{\int_{0}^{\mathrm{t}}\left|\mathrm{A}_{\mathrm{i}}(\mathrm{z})\right|^{2}\left|\mathrm{~A}_{\mathrm{f}}(\mathrm{z})\right|^{2} \mathrm{dz}}$.

In the considered case the measured profile depends on the penetration depth and roughness amplitude. Eq. 8 reduces to the conventional Laplace form [6] for ideal flat surface:

$$
\sigma_{\mathrm{ij}}(\tau)=\frac{\int_{0}^{\mathrm{t}} \exp (-\mathrm{z} / \tau) \sigma_{\mathrm{ij}}(\mathrm{z}) \mathrm{dz}}{\int_{0}^{\mathrm{t}} \exp (-\mathrm{z} / \tau) \mathrm{dz}}
$$

\section{Information depth approximation}

Practical ways for solution of Eq. 8 can be based on least-squares fitting using model functions for $\sigma_{\mathrm{ij}}(\mathrm{z})$ by analogy with the solution of Eq.9 [6]. The simplest one is the information depth approximation. For the fixed angles $\alpha$ and $\beta$ (i.e. fixed penetration depth $\tau$ ) the measured strain is defined then by the strain profile $\sigma_{\mathrm{ij}}(\langle\mathrm{z}\rangle)$ at the depth $\langle\mathrm{z}\rangle=\tau \xi$ with $\xi$ as the dimensionless information depth calculated from the expression [1]: 


$$
\xi=\frac{\int_{0}^{t / \tau} y \exp (-y) d y}{\int_{0}^{t / \tau} \exp (-y) d y} \approx 1, \text { if } t>>\tau .
$$

Using the same assumptions, the expression for the rough surface follows:

$$
\xi(\tau, u)=\frac{\int_{0}^{\infty}\left|A_{i}(\tau y)\right|^{2}\left|A_{f}(\tau y)\right|^{2} y d y}{\int_{0}^{t}\left|A_{i}(\tau y)\right|^{2}\left|A_{f}(\tau y)\right|^{2} d y},
$$

and thus the dimensionless information depth depends on the ratio between the penetration depth and roughness amplitude. Fig. 2 shows several examples of this dependence.

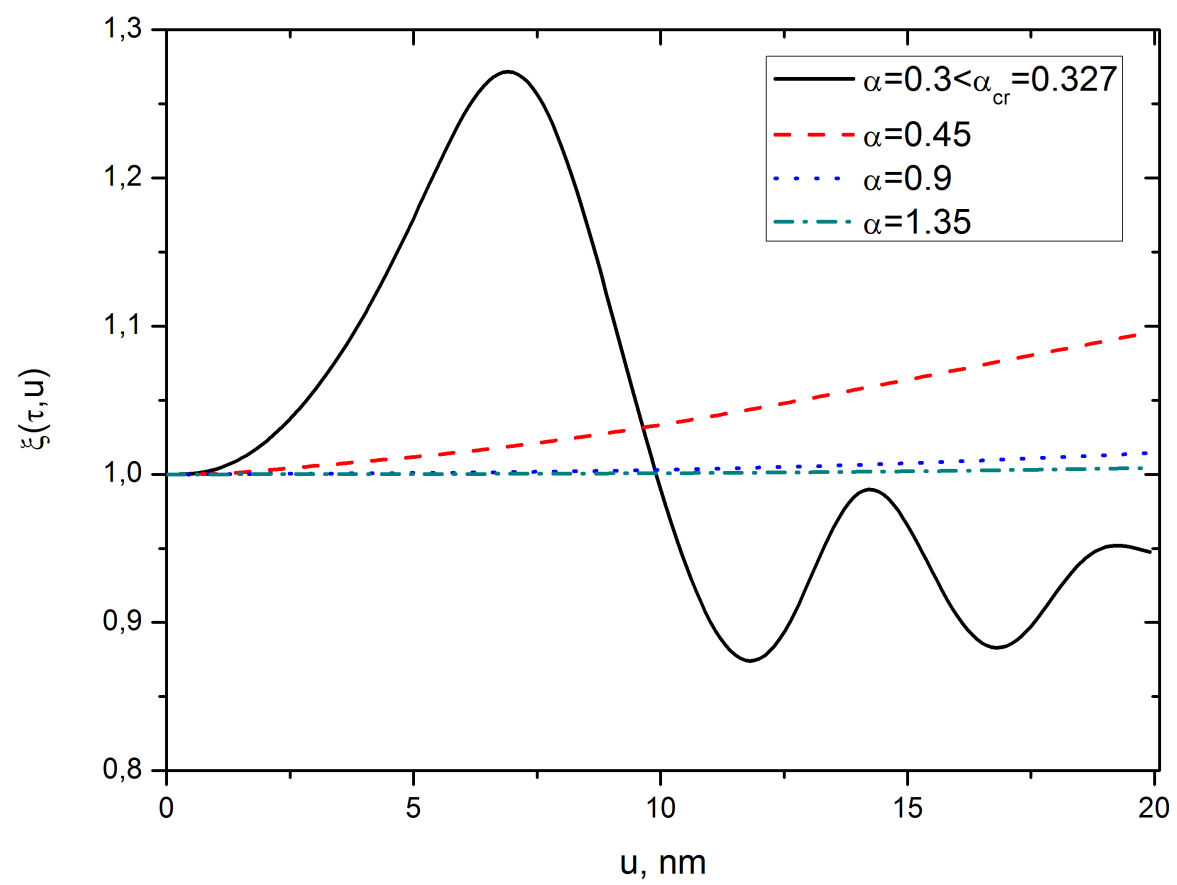

Figure 2

Dimensionless information depth is represented as the function of the roughness amplitude $u$ for the $\mathrm{Ti}_{\mathrm{x}} \mathrm{Cr}_{1-\mathrm{x}} \mathrm{N}$ coating on iron [8]. Minimal penetration depth $\tau=44 \mathrm{~nm}$ corresponds to the reflection (111) with the incident angle $\alpha=0.45^{\circ}$. As for example, value $\alpha$ less than the critical angle is also considered in order to demonstrate more complicated dependence $\xi(u)$ in this case.

The dependence of the information depth on the roughness influences the stress gradient evaluation results. Fig. 3 compares the results of the stress calculation in $\operatorname{Ti}_{x} \mathrm{Cr}_{1-\mathrm{x}} \mathrm{N}$ coating [8] obtained for the model of ideal and rough surfaces. Roughness amplitude $u=14 \mathrm{~nm}$ was found from the reflectivity profile and the strain was measured by multiple (hkl) method [6]. The shift of the information depth, however, is not essential for the considered sample because the condition (2) is not fulfilled. As for example, $u \mu / \alpha \approx 1 / 3$ for the Bragg reflection (111) and information depth 
increases $\approx 5 \%$ as one can see from Fig. 2 . The same value defines the relative shift of the calculated stress profile at Fig. 3 if the roughness is taken into account.

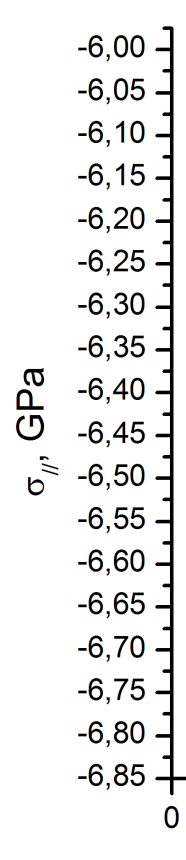

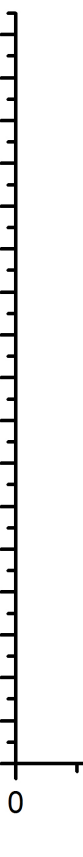




\section{References}

[1] A.Kumar, U.Welzel and E.J.Mittemeijer: J. Appl. Cryst. Vol. 39 (2006), p.633

[2] M.H.Ott and D.Löhe: Advances in X-ray Analysis, Vol. 46 (2003), p. 232

[3] Ch.Genzel: Phys. Status Solidi A Vol. 159 (1997), p. 283

[4] U.Pietsch, V.Holy and T.Baumbach: X-Ray Scattering: from Thin Films to Lateral Nanostructure (Springer-Verlag, Heidelberg 2004).

[5] I.Feranchuk, S.Feranchuk, L.Komarov, S.Sytova and A.Ulyanenkov: Phys. Rev. B Vol. 67 (2003), p.235417

[6] U.Welzel, J.Ligot, P.Lamparter, A.S.Vermeulen and E.J.Mittemeijer: J. Appl. Cryst. Vol. 38 (2005), p.1

[7] H.Dölle: J. Appl. Cryst. Vol. 12 (1979), p.489

[8] V.Uglov, V.Anischik, S.Zlotski, I.Feranchuk, T.Alexeeva, A.Ulyanenkov, J.Brechbuehl and A.Lazar: Surface and Coating Technology. Vol. 202 (2008), p.2389 